\title{
Internal and Emergency Medicine from a 2016 perspective
}

\author{
Domenico Prisco ${ }^{1}$
}

Published online: 21 March 2016

(c) SIMI 2016

Dear Readers,

Internal and Emergency Medicine has entered into the 11th year of publication.

In 2015 we published 194 papers, with an average increase of $39 \%$ over 2014 . We have emphasized the publication of original contributions. The time from acceptance to publication is usually less than 12 months. The chairman of the section of Emergency Medicine: Bruce Adams has expended a great amount of energy and editorial work. He has also renewed the editorial board of the Emergency Medicine section, and has appointed several new associate editors. Interestingly the Emergency Medicine section is very active, and receives an increasing number of submissions from all over the world, now approaching the figures of the Internal Medicine section. In 2015 we counted approximately 130 original articles submitted to the Emergency Medicine section.
The Health Technology Assessment and Clinical Evidence section is also growing thanks to the work of Giorgio Costantino and Giovanni Casazza together with all the other editors.

Data from the Publisher confirm a $16 \%$ increase of Full Text Downloads of our papers from 2014 to 2015. In 2015 we counted a total of 93,185 downloads.

Truly the success of our Journal is also demonstrated by the number of submissions in January and February 2016, which were 109! This means about 55 papers per month versus the 40 papers per month we reached in 2015.

Thanks to all the members of Editorial Board for their work!

We hope you continue to enjoy and support our Journal, and we wish you a wonderful 2016 !

Domenico Prisco

domenico.prisco@unifi.it

1 Interdisciplinary Internal Medicine Unit, University of Florence, AOU Careggi, Florence, Italy 